\title{
Determination of the far-infrared dust opacity in a prestellar core ${ }^{\star}$
}

\author{
A. Suutarinen ${ }^{1,2}$, L. K. Haikala ${ }^{3,2}$, J. Harju ${ }^{3,2}$, M. Juvela ${ }^{2}$, Ph. André ${ }^{4}$, J. M. Kirk ${ }^{5}$, V. Könyves ${ }^{4,6}$, and G. J. White ${ }^{1,7}$ \\ 1 Department of Physics and Astronomy, The Open University, Walton Hall Milton Keynes, MK7 6AA, UK \\ e-mail: aleksi.suutarinen@gmail.com \\ 2 Department of Physics, University of Helsinki, PO Box 64, 00014 Helsinki, Finland \\ 3 Finnish Centre for Astronomy with ESO, University of Turku, Väisäläntie 20, 21500 Piikkiö, Finland \\ ${ }^{4}$ Laboratoire AIM, CEA/DSM-CNRS-Université Paris Diderot, IRFU/Service d'Astrophysique, CEA Saclay, Orme des Merisiers, \\ 91191 Gif-sur-Yvette, France \\ 5 Jeremiah Horrocks Institute, University of Central Lancashire, Preston PR1 2HE, UK \\ ${ }^{6}$ Institut d'Astrophysique Spatiale, UMR 8617, CNRS/Université Paris-Sud 11, 91405 Orsay, France \\ 7 RAL Space, STFC Rutherford Appleton Laboratory, Chilton Didcot, Oxfordshire OX11 0QX, UK
}

Received 23 February 2012 / Accepted 11 June 2013

\section{ABSTRACT}

\begin{abstract}
Context. Mass estimates of interstellar clouds from far-infrared and submillimetre mappings depend on the assumed dust absorption cross-section for radiation at those wavelengths.

Aims. The aim is to determine the far-IR dust absorption cross-section in a starless, dense core located in Corona Australis. The value is needed for determining of the core mass and other physical properties. It can also have a bearing on the evolutionary stage of the core.

Methods. We correlated near-infrared stellar $H-K_{\mathrm{s}}$ colour excesses of background stars from NTT/SOFI with the far-IR optical depth map, $\tau_{\mathrm{FIR}}$, derived from Herschel 160, 250, 350, and $500 \mu \mathrm{m}$ data. The Herschel maps were also used to construct a model for the cloud to examine the effect of temperature gradients on the estimated optical depths and dust absorption cross-sections.

Results. A linear correlation is seen between the colour $H-K_{\mathrm{S}}$ and $\tau_{\mathrm{FIR}}$ up to high extinctions $\left(A_{V} \sim 25\right)$. The correlation translates to the average extinction ratio $A_{250 \mu \mathrm{m}} / A_{J}=0.0014 \pm 0.0002$, assuming a standard near-infrared extinction law and a dust emissivity index $\beta=2$. Using an empirical $N_{\mathrm{H}} / A_{J}$ ratio we obtain an average absorption cross-section per $\mathrm{H}$ nucleus of $\sigma_{250 \mu \mathrm{m}}^{\mathrm{H}}=(1.8 \pm 0.3) \times 10^{-25} \mathrm{~cm}^{2} \mathrm{H}$-atom ${ }^{-1}$, corresponding to a cross-section per unit mass of gas $\kappa_{250 \mu \mathrm{m}}^{\mathrm{g}}=0.08 \pm 0.01 \mathrm{~cm}^{2} \mathrm{~g}^{-1}$. The cloud model, however, suggests that owing to the bias caused by temperature changes along the line-of-sight, these values underestimate the true cross-sections by up to $40 \%$ near the centre of the core. Assuming that the model describes the effect of the temperature variation on $\tau_{\mathrm{FIR}}$ correctly, we find that the relationship between $H-K_{\mathrm{S}}$ and $\tau_{\mathrm{FIR}}$ agrees with the recently determined relationship between $\sigma^{\mathrm{H}}$ and $N_{\mathrm{H}}$ in Orion A.

Conclusions. The derived far-IR cross-section agrees with previous determinations in molecular clouds with moderate column densities, and is not particularly large compared with some other cold cores. We suggest that this is connected to the core not being very dense (the central density is likely to be $\sim 10^{5} \mathrm{~cm}^{-3}$ ), and judging from previous molecular line data, it appears to be at an early stage of chemical evolution.
\end{abstract}

Key words. ISM: clouds - dust, extinction

\section{Introduction}

Determining the emission properties of interstellar dust is useful not only for providing reliable molecular cloud mass estimates from thermal dust emission maps, but also for testing ideas about dust evolution. In several previous studies the far-infrared to visual or far-IR to near-IR extinction ratio $\left(A_{\lambda} / A_{V}\right.$ or $\left.A_{\lambda} / A_{J}\right)$, sometimes called the emissivity, has been studied by combining near-infrared photometry and dust continuum maps (Bianchi et al. 2003; Kramer et al. 2003; Shirley et al. 2005; Lehtinen et al. 2007; Shirley et al. 2011). One of the results from these studies is that the dust emissivity changes from region to region, and it shows a tendency to increase with decreasing dust temperature (Arab et al. 2012). This trend agrees well with theoretical predictions, is thought to be caused primarily by dust coagulation in the cold, dense cores of molecular clouds, and is further accentuated by ice formation on the surfaces of dust grains

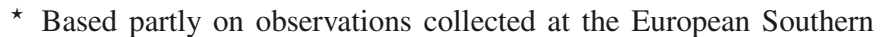
Observatory, Chile (ESO programmes 075.C-0748 and 077.C-0562).
(Ossenkopf \& Henning 1994; Stepnik et al. 2003; Paradis et al. 2009; Ormel et al. 2011).

The availability of multi-frequency data from the Planck and Herschel satellites has vastly improved the accuracy of the determination of dust properties and physical conditions in molecular clouds. In particular, the wavelengths accessible to Herschel cover both sides of the emission maximum of cold $(T \sim 10 \mathrm{~K})$ interstellar dust. Earlier surveys usually had only two or three frequencies in the submillimetre/far-infrared and often only on one side of the peak.

In this paper we determine the far-infrared dust emissivity and absorption cross-section in a prestellar core using sensitive near-infrared photometry in the $H$ and $K_{\mathrm{s}}$ bands in conjunction with Herschel maps at four wavelengths. In this way we believe we can achieve higher accuracy than previous studies have. Furthermore, the target core provides a useful reference because it has been claimed that it represents a very early stage of chemical evolution where molecular depletion is not significant (Kontinen et al. 2003). The core, which lies in the "tail" of the Corona Australis molecular cloud, can be found in the 
Planck Early Release Compact Source Catalogue as PLCKECC G359.78-18.34", and has been previously called "CrA C" (Harju et al. 1993) and "SMM 25" (Chini et al. 2003). So far, no indication of star formation taking place in $\mathrm{CrA} \mathrm{C}$ has been found in the millimetre continuum (Chini et al. 2003), in submillimetre (Herscel), or in the mid- and near-infrared (Peterson et al. 2011).

In Sect. 2 we briefly discuss some aspects of $J H K$ photometry towards highly reddened stars, and in Sect. 3 we describe the near- and far-infrared observations used in the present work. In Sect. 4, the near-IR reddening is correlated with the far-IR optical depth, and the result is used to derive the dust absorption cross-section. Finally, in Sect. 5 we discuss the significance of the obtained results.

\section{On JHK photometry at high extinctions}

Analysis of $J H K$ near-infrared photometry involves some uncertainties and complications. The $J H K$ system is not uniquely defined because different detectors and filter sets have been used. Additionally the transmissions in the $J H K$ bands depend strongly on the atmospheric transmission (atmospheric $\mathrm{H}_{2} \mathrm{O}$ absorption lines) and thus vary depending on the observing site and is even subject to night-to-night variations. As a consequence one should be careful when comparing photometric data obtained at different epochs and observing sites. This is also true when adapting a $J H K$ reddening slope because these slopes depend on the particular filter-detector combination. The modified $K_{\mathrm{s}}$ is narrower than the $K$ filter and excludes some of the strong atmospheric lines and is thus less influenced by the atmospheric opacity.

The sensitivity of near-infrared observations has increased dramatically during the last decade, and it is now possible to observe routinely highly reddened, faint stars. The reddening affects the observed object spectral energy distribution (SED) and for highly reddened sources the effective wavelength of the filter shifts towards longer wavelengths. This introduces a new effect as the $J H K$ reddening slope changes depending on the reddening. This phenomenon was already noted in the $U B V$ system (see e.g. Golay 1974), and has been later studied e.g. using the Two Micron All Sky Survey (2MASS) ${ }^{2}$ (Straižys \& Lazauskaite 2008), and the UKIRT Infrared Deep Sky Survey (UKIDSS) data (Stead \& Hoare 2009). As noted in Stead \& Hoare (2009) one should refer to a reddening track instead of a reddening slope. The importance of the photometric system is highlighted by the fact that in the 2MASS system the reddening track of highly reddened giants curves down in the $J H K$ colour-colour diagram whereas in the MKO system (UKIDSS) the track curves up. In addition to reddening this effect depends on the spectral type of the source. The effect is noticeable for $H-K$ indices larger than $\sim 1.5$. In the present work the highest $H-K_{\mathrm{s}}$ indices are nearly 3 .

\section{Data acquisition}

\subsection{NTT/SOFI observations}

The dense core region of CrA C was imaged in $J, H$, and $K_{\mathrm{s}}$ using the Son Of Isaac (SOFI) near-infrared instrument on the New Technology Telescope (NTT) at the La Silla observatory in May 2005 and July 2006. The SOFI field of view is 4!9

\footnotetext{
1 http://irsa.ipac.caltech.edu/applications/planck/

2 http://irsa.ipac. caltech.edu/Missions/2mass.html
}

and the pixel size is 0 !'288. The observing was done using the standard jittering mode in observing blocks of approximately $1 \mathrm{~h}$. Standard stars from the faint near-IR standard list of Persson et al. (1998) were observed frequently during the nights. Because very few stars were seen in the first $J$ band observing block the observations were continued only in the $H$ and $K_{\mathrm{s}}$ bands. The total on source time was $40 \mathrm{~min}, 104 \mathrm{~min}$ and $540 \mathrm{~min}$ in the $J, H$, and $K_{\mathrm{s}}$ filters, respectively. The average seeing was $\sim 0$ ' $^{\prime} 7$.

The $\mathrm{IRAF}^{3}$ external package XDIMSUM was used to reduce the imaging data. The images were searched for cosmic rays and sky-subtracted. The two nearest background images in time to each image were used in the sky subtraction. An object mask was constructed for each image. Applying these masks in the sky subtraction produced hole masks for each sky-subtracted image. Special dome flats and illumination correction frames provided by the NTT team were used to flat field and to illumination correct the sky-subtracted SOFI images. Rejection masks combined from a bad pixel mask and individual cosmic ray and hole masks were used when averaging the registered images. The coordinates of the registered images were derived from the 2MASS catalogue (Skrutskie et al. 2006).

The SExtractor software v 2.5.0 (Bertin \& Arnouts 1996) package was used to obtain stellar photometry of the reduced SOFI images. Galaxies were excluded using the SExtractor CLASS keyword and by visual inspection of the images.

The zero points of the coadded data were fixed using standard star measurements and checked using stars in common with the less deep HAWK-I imaging of CrA reported in Sicilia-Aguilar et al. (2011) (see below). The limiting magnitudes (for a formal error of $0.1 \mathrm{mag}$ ) are approximately 21.5 , $20 \mathrm{~m} .5$, and $20^{\mathrm{m}} 8$ for $J, H$, and $K_{\mathrm{s}}$, respectively. After elimination of the nonstellar sources 25, 266, and 369 stars remained in $J$, $H$, and $K_{\mathrm{s}}$, respectively. In the subsequent analysis we use the 266 stars detected both in the $H$ and $K_{\mathrm{s}}$ filters. The locations of these stars are indicated with blue plus signs in Fig. 4. As one can see in this Figure, no stars were detected in the very centre of CrA C. All detections lie at the edges of the core.

The extracted $J H K_{\mathrm{s}}$ magnitudes were converted into Persson magnitudes, and from these to the 2MASS photometric system as described in Ascenso et al. (2007). However, these conversion formulae could not be directly applied to all of the SOFI data because for most stars $J$ band magnitudes were not available owing to the high extinction. Using the Eqs. (1)-(6) of Ascenso et al. (2007) the conversion formula for the $\left(H-K_{\mathrm{s}}\right)$ index from the instrumental magnitudes to the $2 \mathrm{MASS}$ system can be written as

$\left(H-K_{\mathrm{s}}\right)_{2 \mathrm{MASS}}=1.019\left(H-K_{\mathrm{s}}\right)-0.046\left(J-K_{\mathrm{s}}\right)+0.005$,

where the magnitudes on the right are SOFI magnitudes. We have attempted to decrease the systematic error arising from the missing $J$ magnitude by estimating the $\left(J-K_{\mathrm{s}}\right)$ colour using the observed $H-K_{\mathrm{s}}$ index, the intrinsic $J-K$ and $H-K$ colours of giant stars from Bessell \& Brett (1988), and the extinction ratios $A(\lambda) / A\left(K_{\mathrm{s}}\right)$ derived by Indebetouw et al. (2005). These give

$$
\begin{aligned}
\left(J-K_{\mathrm{s}}\right)_{\mathrm{est}} & =\left(J-K_{\mathrm{s}}\right)_{0}-\frac{E\left(J-K_{\mathrm{s}}\right)}{E\left(H-K_{\mathrm{s}}\right)}\left(H-K_{\mathrm{s}}\right)_{0}+\frac{E\left(J-K_{\mathrm{s}}\right)}{E\left(H-K_{\mathrm{s}}\right)}\left(H-K_{\mathrm{s}}\right) \\
& =(0.39 \pm 0.11)+(2.72 \pm 0.48) \times\left(H-K_{\mathrm{s}}\right) .
\end{aligned}
$$

3 IRAF is distributed by the National Optical Astronomy Observatories, which are operated by the Association of Universities for Research in Astronomy, Inc., under cooperative agreement with the National Science Foundations. 
The ratio of colour excesses $E\left(J-K_{\mathrm{s}}\right) / E\left(H-K_{\mathrm{s}}\right)=2.72 \pm 0.48$ is obtained using the average $A(J) / A\left(K_{\mathrm{s}}\right)$ and $A(H) / A\left(K_{\mathrm{s}}\right)$ values listed in Table 1 of Indebetouw et al. (2005). The corresponding values of $(J-K)_{0}-\frac{E\left(J-K_{\mathrm{s}}\right)}{E\left(H-K_{\mathrm{s}}\right)}(H-K)_{0}$ range from 0.27 to 0.50 for giants of the types G0 to M7 using the intrinsic colours given in Bessell \& Brett (1988). The possible variation of the intrinsic colours combined with the uncertainty of the colour excess ratio causes an additional uncertainty of $\sim 0$. 03 to the derived $H-K_{\mathrm{s}}$ index. This small uncertainty is added in quadrature to the uncertainties of $H-K_{\mathrm{s}}$ owing to formal photometric errors $(\sim 0.09)$ and the uncertainty in the magnitude zero-point $\left(\sim 0\right.$. 07 for $\left.H-K_{\mathrm{s}}\right)$.

The conversion to the 2MASS system using Eqs. (1) and (2) reduces the instrumental $H-K_{\mathrm{s}}$ indices by about 10 percent. The observed $H-K_{\mathrm{s}}$ indices for the highly reddened stars in our sample range from 1.5 to 2 . 9 , and for these stars the correction lies between -0 . 14 and -0 . 25 . Ignoring the colour-dependent term in the conversion to the 2MASS system would bias systematically the $H-K_{\mathrm{s}}$ indices used in the following analysis.

\subsection{VLT/HAWK-I observations}

The core CrA C was partially covered by two 7.5 × 7.5 fields taken with the HAWK-I IR camera on the VLT as part of the survey of Corona Australis reported in Sicilia-Aguilar et al. (2011) (ESO program 083.C-0079). A more detailed description of the observations and reduction procedures can be found in that paper. The photometry zero point of the HAWK-I data was fixed to 2 MASS objects present in the fields. The typical calibration errors range from $1 \%$ to $5 \%$, depending on the weather conditions. Therefore, signal to noise and background remain the main sources of uncertainty, especially for the fainter objects. The data are complete in the following dynamical ranges: $J=11-18.5 \mathrm{mag}, H=11-18.5 \mathrm{mag}, K=10.5-18 \mathrm{mag}$. The locations of HAWK-I stars with reasonably small errors $(<0.1$ in $H$ and $K$ ) lying within a radial distance of 5.5 from the core centre are marked with red crosses in Fig. 4. The total number of these stars is 964 .

Of the 266 stars in our SOFI sample 109 were detected with HAWK-I. In Fig. 1 we correlate the $H-K_{\mathrm{s}}$ indices of stars common to both surveys. Only a zero-point correction to the 2MASS system has been done to the magnitudes presented in this figure. This is because no colour-dependent correction has been applied to the HAWK-I data (Aurora Sicilia-Aquilar, priv. comm.) as the characteristics of the HAWK-I filters needed for this correction are not available. One can see the uncorrected SOFI colours agree with the HAWK-I data. After the colour correction for SOFI, the slope of the correlation deviates clearly from unity because this correction reduces the $H-K_{\mathrm{s}}$ indices by about $10 \%$. Because our main purpose is to derive the dust opacity towards the core where the colour correction has a noticeable effect, we have decided not to use the HAWK-I data in the subsequent analysis.

\section{3. $2 M A S S$ data}

Of the stars detected in the 2MASS survey 58 (again, including only those with $\Delta K$ and $\Delta H<0$. 1 ) lie within a distance of 5.5 from the core centre. The positions of these stars (excluding those coincident with HAWK-I or SOFI stars) are marked in Fig. 4. Only two of the 2MASS stars coincide with those in the SOFI sample. The limiting magnitudes of the 2MASS data are $15.8,15^{\mathrm{m}} \cdot 1$, and 14.2 in $J, H$, and $K$, respectively. Because the 2MASS data clearly probe the diffuse envelope around the

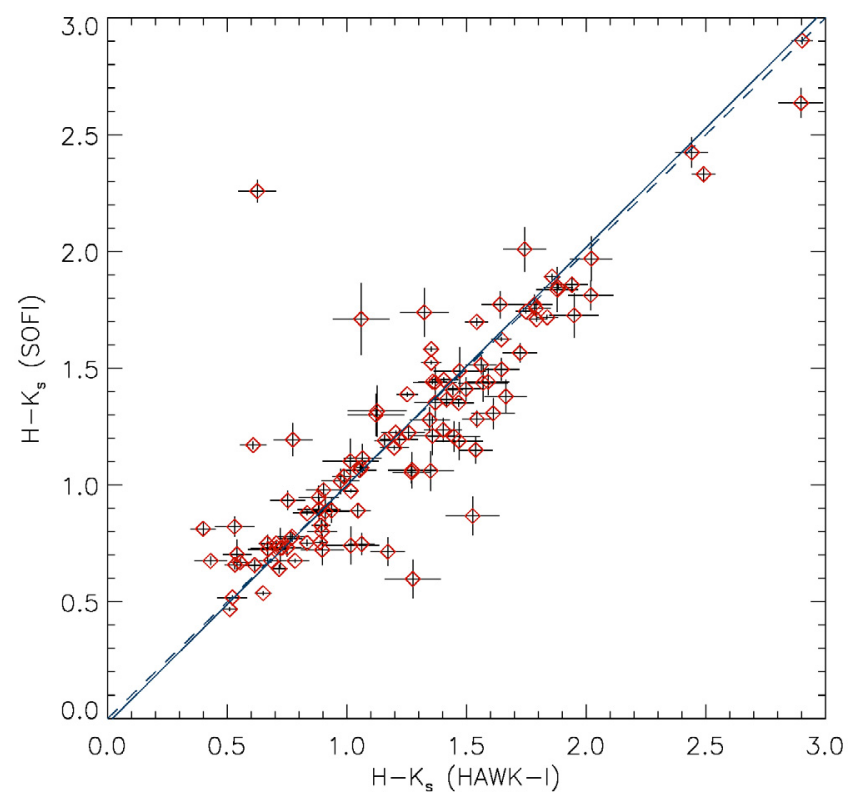

Fig. 1. Relationship between the $H-K_{\mathrm{s}}$ colours of the 109 stars detected with both SOFI and HAWK-I, before performing the colour-dependent conversion to the 2 MASS system. The photometric errors of the data points are indicated. The solid blue line shows the fit to the data, and the dashed line represents the one-to-one correlation.

core we will not use them in the derivation of the submillimetre opacity.

\subsection{Herschel data}

The $15^{\prime} \times 15^{\prime}$ far-IR and submillimetre maps of CrA $\mathrm{C}$ used in this study were extracted from an extensive mapping of the Corona Australis region made as part of the Herschel Gould Belt Survey (André et al. 2010) with the SPIRE (Griffin et al. 2010) and PACS (Poglitsch et al. 2010) instruments onboard Herschel (Pilbratt et al. 2010). The original CrA maps cover a region of about $5^{\circ} \times 2^{\circ}$ at the wavelengths 70 and $160 \mu \mathrm{m}$ (PACS) and 250, 350, and $500 \mu \mathrm{m}$ (SPIRE). The SPIRE observations were reduced with HIPE 7.0, using modified pipeline scripts, then, the default naive mapping routine was applied to produce the final maps. PACS data was processed within HIPE 8.0 up to level 1 after which we employed Scanamorphos v16 (Roussel 2012) to create the map products. The maps of the whole $\mathrm{CrA}$ field will become available on the Herschel Gould Belt Survey Archives ${ }^{4}$. The beam FWHMs for each wavelength are $36^{\prime \prime}, 25^{\prime \prime}, 18^{\prime \prime}$, $12^{\prime \prime} \times 16^{\prime \prime}$, and $6^{\prime \prime} \times 12^{\prime \prime}$ for $500,350,250,160$, and $70 \mu \mathrm{m}$, respectively, the PACS beams being clearly elongated in observations carried out in the parallel mode. The Herschel intensity map at $\lambda=250 \mu \mathrm{m}, I_{250 \mu \mathrm{m}}$, is shown in Fig. 2.

Based on comparison with the Planck satellite maps of the region the following offsets were added to the Herschel maps from 70 to $500 \mu \mathrm{m}$, respectively: $2.5,14.9,15.6,8.7$, 4.0 MJy/sr. The maps at $160,250,350$, and $500 \mu \mathrm{m}$ were convolved to a resolution of $40^{\prime \prime}$ (FWHM), and the intensity distributions were fitted with a modified blackbody function, $I_{v} \approx B_{v}\left(T_{\text {dust }}\right) \tau_{v} \propto B_{v}\left(T_{\text {dust }}\right) v^{\beta}$, which characterises optically thin thermal dust emission at far-IR and submillimetre wavelengths. We fixed the emissivity/opacity exponent to $\beta=2.0$. The resulting $T_{\text {dust }}$ map is shown in Fig. 3. The distribution of the optical depth, $\tau(250 \mu \mathrm{m})=I_{250 \mu \mathrm{m}} / B_{250 \mu \mathrm{m}}\left(T_{\text {dust }}\right)$, is shown

4 http://gouldbelt-herschel.cea.fr/archives 


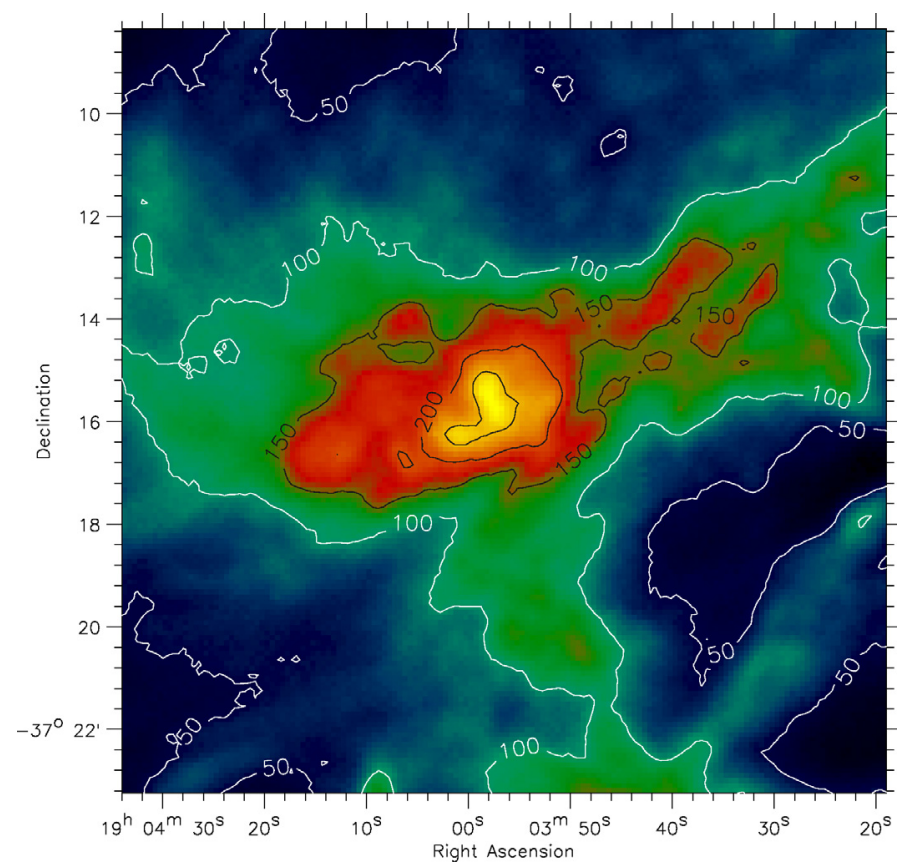

Fig. 2. Herschel $\lambda=250 \mu \mathrm{m}$ map of $\mathrm{CrA} \mathrm{C}$. The contours indicate the intensity $I_{250} \mu \mathrm{m}$ levels ranging from 50 to $250 \mathrm{MJy} / \mathrm{sr}$. The FWHM of the Herschel beam at this wavelength is $18^{\prime \prime}$.

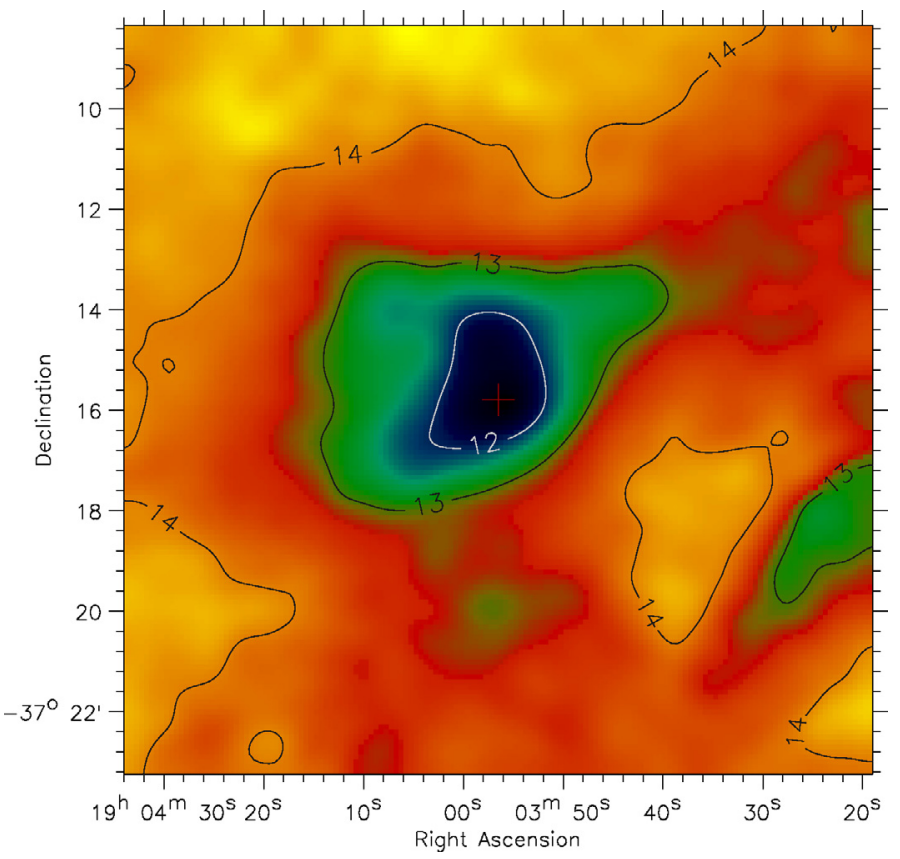

Fig. 3. Dust temperature ( $T_{\mathrm{D}}$ in $\left.\mathrm{K}\right)$ map of CrA C derived from Herschel data.

in Fig. 4. On this map we have plotted the SOFI stars selected for extinction estimates (see below), and the 2MASS and HAWK-I stars within a radius of 5.5 of the core centre.

The errors of $\tau_{250 \mu \mathrm{m}}$ were estimated from a Monte Carlo method using the $1 \sigma$ error maps provided for the three SPIRE bands and a 7\% uncertainty of the absolute calibration for all four bands according to the information given in SPIRE and PACS manuals (SPIRE Observers' Manual, Version 2.4; PACS Observer's Manual, Version 2.4). Different realisations of the $\tau_{250} \mu \mathrm{m}$ map were calculated by combining the four intensity maps with the corresponding error maps, assuming that the error in each pixel is normally distributed. The $\tau_{250 \mu \mathrm{m}}$ error in each pixel was obtained from the standard deviation of one thousand realisations.

\section{Data analysis and results}

As we are dealing with a dense core with high near-infrared extinction the number of background stars with accurate $J$ magnitudes is low. To obtain reasonably good statistics, and to extend extinction estimates close to the core centre, we have only used the $H$ and $K_{\mathrm{s}}$ bands where the extinction is less severe than in $J$. A further motivation for the use of $H$ and $K_{\mathrm{s}}$ is the fact that the intrinsic $H-K$ colours of giants, the class of the background stars most probably observed towards the cloud span a rather narrow range of $(H-K)_{0}=0.07-0.31 \mathrm{mag}$ (Bessell \& Brett 1988). The stellar near-infrared colour excess $E(H-K) \equiv(H-K)-(H-K)_{0}$ can be used to evaluate the dust column density (Lada et al. 1994).

Because of the reasons discussed in Sects. 2.1-2.3, the HAWK-I and 2MASS stars were used only for checking the zero point of the magnitude scale for the SOFI stars, but not in the estimation of the dust opacity in the CrA $\mathrm{C}$ core. For this purpose we used SOFI stars with photometric $1-\sigma$ errors of $\Delta H<0.1 \mathrm{mag}, \Delta K<0.1 \mathrm{mag}$. The number of stars fulfilling these criteria is 266. The locations of these stars are indicated with white plus signs in the Herschel far-IR optical depth $\left(\tau_{250 \mu \mathrm{m}}\right)$ map shown in Fig. 4.

Besides the colour excess $E\left(H-K_{\mathrm{s}}\right)$ also the optical depth $\tau_{250 \mu}$ m measures the column density of dust, albeit at very different angular resolution. We have tried to reconcile this difference in the following way: from the Herschel map we have only taken those pixels that contain one or more background stars. The $H-K_{\mathrm{s}}$ value assigned to this pixel is then calculated as the average of the neighbouring stars weighted by a $40^{\prime \prime}$ Gaussian beam to match the resolution of the smoothed dust emission maps. The $H-K_{\mathrm{s}}$ measurements for individual stars taken in the averages were furthermore weighted according to their errors owing to the photometric noise and to the uncertainty of the $J-K_{\mathrm{s}}$ colour needed for the conversion to the 2MASS system as discussed in Sect. 3.1. The errors of the $H-K_{\mathrm{s}}$ values corresponding to pixels in the $\tau_{250 \mu \mathrm{m}}$ map were calculated using the standard formula for a weighted average. The resulting correlation plot $H-K_{\mathrm{S}}$ vs. $\tau(250 \mu \mathrm{m})$ is shown in Fig. 5. The typical (mean) error for the colours of the SOFI stars is indicated. The horizontal error bar represents the mean error of the $\tau_{250 \mu \mathrm{m}}$ estimates (see Sect. 2.4).

A linear fit to the SOFI data gives the following model (shown in Fig. 5):

$$
H-K_{\mathrm{s}}=(0.14 \pm 0.02)+(174 \pm 4) \tau_{250 \mu \mathrm{m}} \text {. }
$$

The point where the model intersects the $y$-axis should represent the average intrinsic colour $\left(H-K_{\mathrm{s}}\right)_{0}$ of the background stars. This assumption seems reasonable since the intersection, $0 .{ }^{\mathrm{m}} 14$, corresponds to the intrinsic $H-K$ colour of a $\mathrm{K}$ type giant (Bessell \& Brett 1988). Therefore, converting the far-infrared optical depth to extinction, the fit result can be written as $A(250 \mu \mathrm{m})=(0.0062 \pm 0.0001) E\left(H-K_{\mathrm{s}}\right)$. Here we have used the relationship $A_{250 \mu \mathrm{m}}=1.086 \tau_{250 \mu \mathrm{m}}$.

The colour excess can be converted to the near-infrared extinction, e.g. $A(J)$, using the wavelength dependence of extinction. According to the extinction ratios, $A(J) / A\left(K_{\mathrm{s}}\right)=2.50 \pm$ 0.15 and $A(H) / A\left(K_{\mathrm{s}}\right)=1.55 \pm 0.08$, presented in Table 1 of 
A. Suutarinen et al.: Determination of the far-infrared dust opacity in a prestellar core

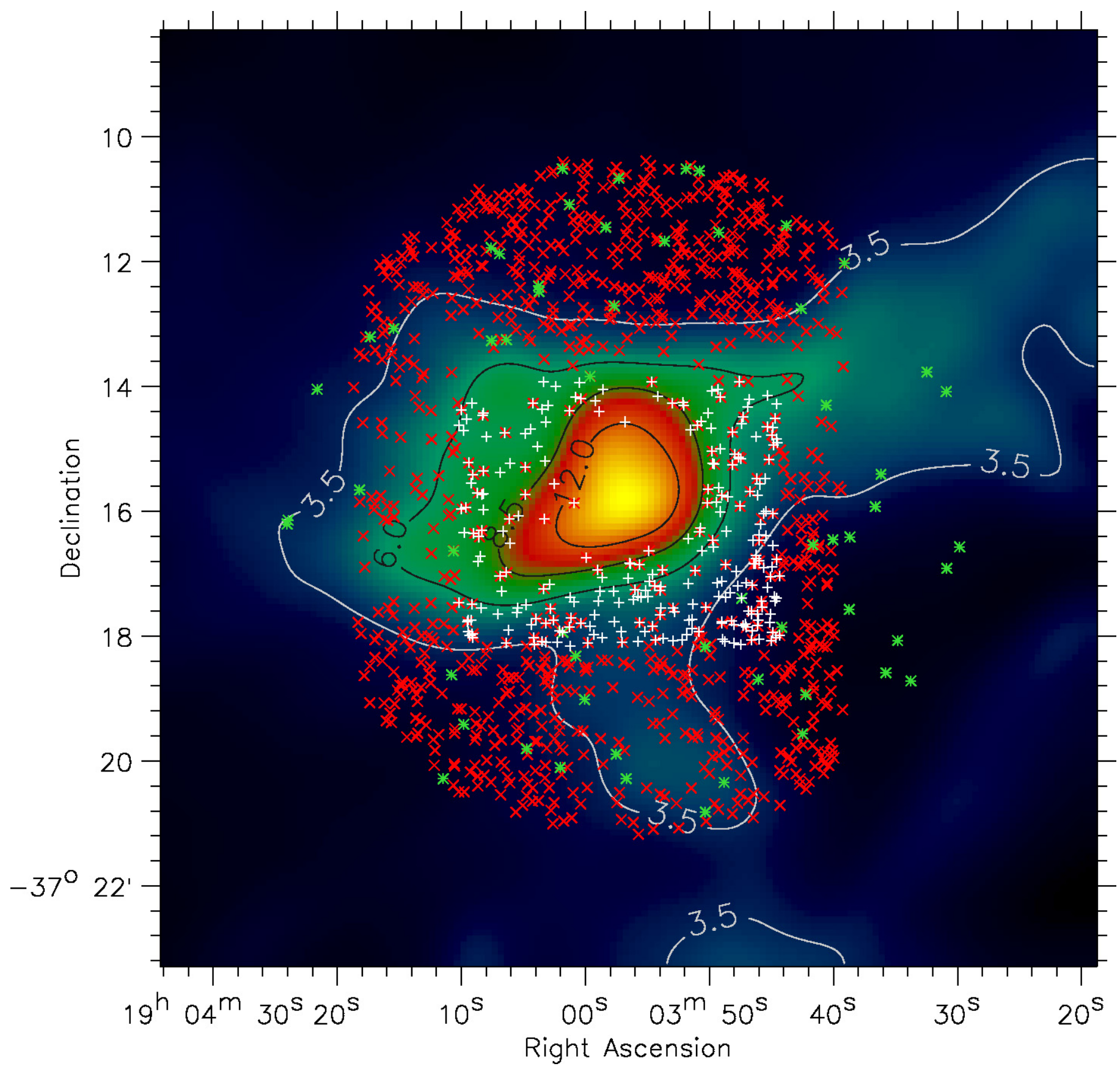

Fig. 4. Locations of background stars on the optical depth $\tau(250 \mu \mathrm{m})$ map from Herschel. The contour labels give $\tau(250 \mu \mathrm{m})$ values multiplied by 1000. Different symbols have been used to distinguish between stars from the three near-IR surveys: 2MASS (green asterisks), HAWK-I (red crosses), and SOFI (white plus signs). Of the HAWK-I and 2MASS stars only those within a 5.5 radius of the map centre are shown here.

Indebetouw et al. (2005) the relationship between $A(J)$ and $E\left(H-K_{\mathrm{s}}\right)$ is $A_{J}=(4.55 \pm 0.72) E\left(H-K_{\mathrm{s}}\right)$. This relationship is consistent with the extinction law from Cardelli et al. (1989), when the wavelengths of the 2MASS $J, H$, and $K_{\mathrm{s}}$ bands are used in their interpolation formula (2a) and (2b), giving $A_{J}=$ $4.44 E\left(H-K_{\mathrm{s}}\right)$. Using the results of Indebetouw et al. (2005) we obtain the following extinction ratio:

$\frac{A_{250 \mu \mathrm{m}}}{A_{J}}=0.0014 \pm 0.0002$.

This extinction ratio is very similar to the value 0.0015 listed in Table 1 of Mathis (1990), and also agrees with the synthetic extinction law based on the dust model developed by
Weingartner \& Draine (2001) and Li \& Draine (2001) $)^{5}$. According to the assumption $\beta=2.0$ used here, the extinction ratio for other wavelengths longer than $160 \mu \mathrm{m}$ can be obtained by multiplying the number above by $(250 \mu \mathrm{m} / \lambda)^{2}$.

To facilitate comparison with some previous results we note that, extrapolating the extinction ratio to the submillimetre regime, our result corresponds to $A_{850 \mu \mathrm{m}} / A\left(K_{\mathrm{S}}\right)=3.0 \times 10^{-4}$ or $A_{850 \mu \mathrm{m}} / A_{V}=3.9 \times 10^{-5}$. In the latter ratio we have assumed that $A_{J} / A_{V}=0.333$ (Cardelli et al. 1989 for $R_{V}=5.5$ ).

\footnotetext{
5 Data available at

http://www . astro.princeton.edu/ draine/dust/dustmix . html
} 


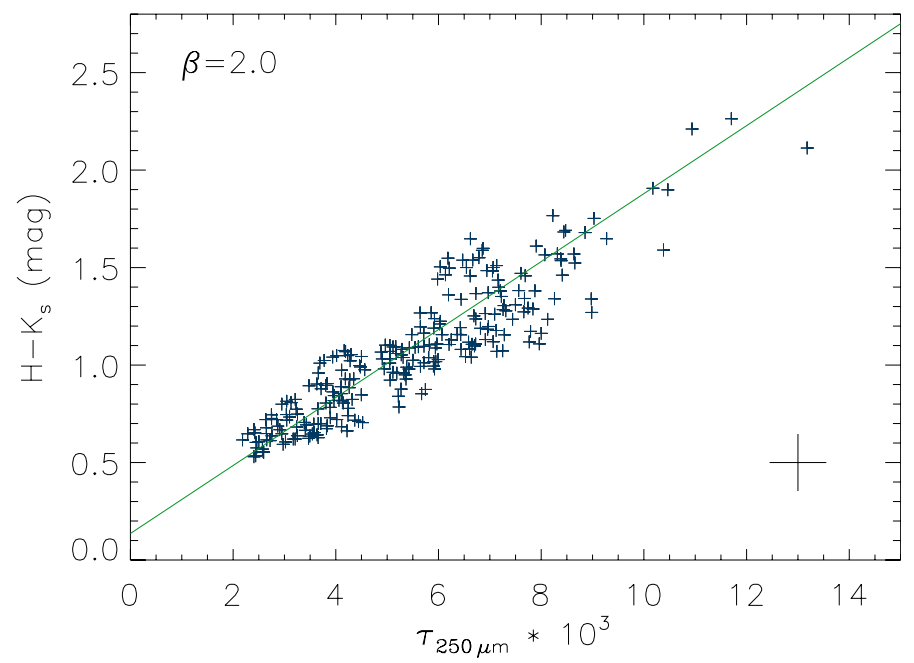

Fig. 5. $H-K_{\mathrm{s}}$ colours of the background stars detected with SOFI as a function of far-IR optical depth of the dust emission. The mean error of the data points is indicated with a cross in the bottom right.

The dust absorption cross-section per $\mathrm{H}$ nucleus, $\sigma_{250 \mu \mathrm{m}}^{\mathrm{H}}$, or per unit mass of gas, $\kappa_{250 \mu \mathrm{m}}^{\mathrm{g}}$, can be estimated using empirical determinations of the $N_{\mathrm{H}} / A_{J}$ or $N_{\mathrm{H}} / A_{V}$ ratio. As discussed by Martin et al. (2012), the ratios of near-infrared colour excess, or alternatively near-infrared extinction to $N_{\mathrm{H}}$ are likely to change less than for example the ratio of $A_{V}$ to $N_{\mathrm{H}}$ when dust grains evolve in dense material. Therefore one could expect that the ratio $N_{\mathrm{H}} / A_{J}$ does not change substantially from diffuse to dense clouds.

The $N_{\mathrm{H}} / E(B-V)$ ratio from the Ly $\alpha$ absorption measurement of Bohlin et al. (1978) with the value $R_{V}=3.1$ characteristic of Galactic diffuse clouds and the corresponding $A_{J} / A_{V}$ ratio $(=0.282)$ give $N_{\mathrm{H}} / A_{J}=6.6 \times 10^{21} \mathrm{~cm}^{-2} \mathrm{mag}^{-1}$. Vuong et al. (2003) determined in the dark cloud $\rho$ Oph $N_{\mathrm{H}} / A_{J}=$ $5.6-7.2 \times 10^{21} \mathrm{~cm}^{-2} \mathrm{mag}^{-1}$ (depending on the assumed metal abundances) by combining X-ray absorption measurements with near-infrared photometry. Recently, Martin et al. (2012, Eq. (9)) compiled a correlation between $N_{\mathrm{H}}$ and $E\left(J-K_{\mathrm{S}}\right)$ from several previous surveys. Their best-fit slope, $N_{\mathrm{H}} / E\left(J-K_{\mathrm{s}}\right)=$ $(11.5 \pm 0.5) \times 10^{21} \mathrm{~cm}^{-2} \mathrm{mag}^{-1}$, together with $A_{J} / E\left(J-K_{\mathrm{s}}\right)=$ $1.67 \pm 0.07$ from Indebetouw et al. (2005) imply the ratio $N_{\mathrm{H}} / A_{J}=(6.9 \pm 0.4) \times 10^{21} \mathrm{~cm}^{-2} \mathrm{mag}^{-1}$. Adopting this $N_{\mathrm{H}} / A_{J} \mathrm{ra}-$ tio we estimate for $\sigma^{\mathrm{H}}$ and $\kappa^{\mathrm{g}}$ the following values:

$$
\begin{aligned}
\sigma_{250 \mu \mathrm{m}}^{\mathrm{H}} & =(1.8 \pm 0.3) \times 10^{-25} \mathrm{~cm}^{2}(\mathrm{H} \text { atom })^{-1}, \\
\kappa_{250 \mu \mathrm{m}}^{\mathrm{g}} & =(0.08 \pm 0.01) \mathrm{cm}^{2} \mathrm{~g}^{-1} \text { of gas, }
\end{aligned}
$$

where $\kappa^{\mathrm{g}}$ is obtained from $\sigma^{\mathrm{H}}$ by dividing it by the average gas particle mass per $\mathrm{H}$ nucleus $\left(=1.4 m_{\mathrm{H}}\right.$, assuming $\left.10 \% \mathrm{He}\right)$. The range of $N_{\mathrm{H}} / A_{J}$ values obtained by Vuong et al. (2003) would imply $\sigma_{250 \mu \mathrm{m}}^{\mathrm{H}}=1.8-2.3 \times 10^{-25} \mathrm{~cm}^{2} \mathrm{H}^{-1}, \kappa_{250 \mu \mathrm{m}}^{\mathrm{g}}=$ $0.08-0.10 \mathrm{~cm}^{2} \mathrm{~g}^{-1}$. Note that $\kappa$ is often given per unit mass of dust in which case the number above should be multiplied by the assumed gas-to-dust mass ratio (usually 100-150).

The values implied by the parameter $C_{250}$ given in Hildebrand (1983) are $\sigma_{250 \mu \mathrm{m}}^{\mathrm{H}}=1.4 m_{\mathrm{H}} / C_{250}=2.3 \times$ $10^{-25} \mathrm{~cm}^{2}(\mathrm{H} \text { atom })^{-1}, \kappa_{250 \mu \mathrm{m}}^{\mathrm{g}}=1 / C_{250}=0.10 \mathrm{~cm}^{2} \mathrm{~g}^{-1}$, that is, slightly larger than those obtained above. Again, $\kappa_{250 \mu \mathrm{m}}^{\mathrm{g}}$ can be converted to other wavelengths by multiplying by $(250 \mu \mathrm{m} / \lambda)^{2}$. For $160 \mu \mathrm{m}$ we obtain $\kappa_{160 \mu \mathrm{m}}^{\mathrm{g}}=(0.19 \pm 0.03) \mathrm{cm}^{2} \mathrm{~g}^{-1}$, and

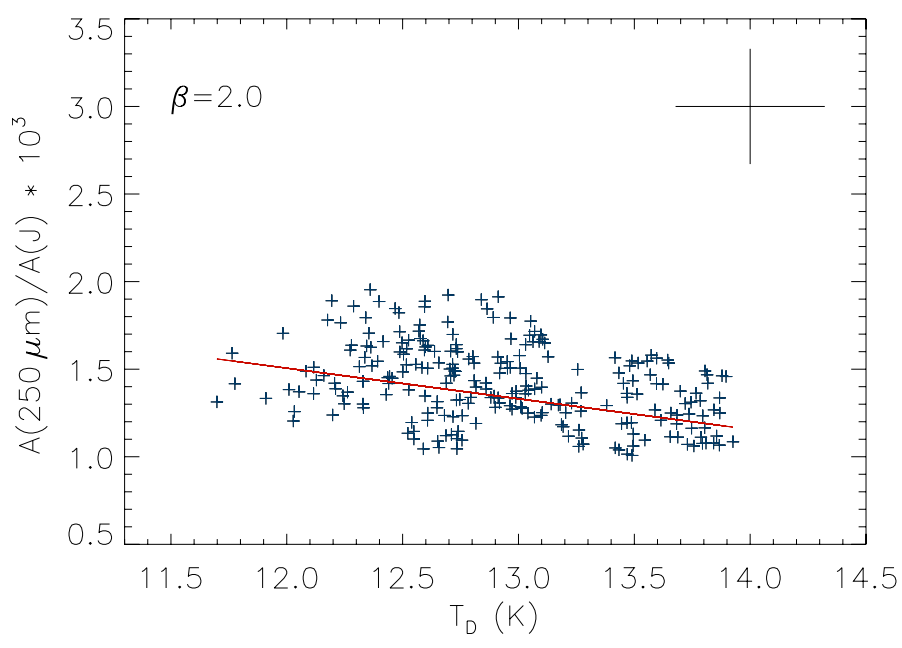

Fig. 6. Extinction ratio $A_{250 \mu \mathrm{m}} / A_{J}$ vs. $T_{\text {dust }}$. The red line represents a linear fit to the data points using SOFI - Herschel comparison (blue crosses). The mean error of the data points is indicated with a cross in the top right.

extrapolating our value to $850 \mu \mathrm{m}$ we get $\kappa_{850 \mu \mathrm{m}}^{\mathrm{g}}=(0.007 \pm$ $0.001) \mathrm{cm}^{2} \mathrm{~g}^{-1}$.

The ratio $A_{250 \mu \mathrm{m}} / A_{J}$ is plotted as a function of $T_{\text {dust }}$ in Fig. 6. One can see that the ratios show a large scatter, but a decreasing tendency towards higher temperatures can be discerned. A linear fit to the data points gives the following relationship $A_{250 \mu \mathrm{m}} / A_{J}=(3.6 \pm 0.5) \times 10^{-3}-(1.7 \pm 0.4) \times 10^{-4} T_{\text {dust }}$, which suggests that the average ratio increases from 0.0012 to $0.0016(30 \%)$ when $T_{\text {dust }}$ decreases from $14 \mathrm{~K}$ to $12 \mathrm{~K}$. Using the previously quoted $N_{H} / A_{J}$ ratio $6.9 \times 10^{21} \mathrm{~cm}^{-2} \mathrm{mag}^{-1}$ this tendency in the extinction ratio can be translated to the dust extinction cross-section: the correlation seems to suggest that $\sigma_{250 \mu \mathrm{m}}^{\mathrm{H}}$ increases from $1.5 \times 10^{-25}$ to $2.0 \times 10^{-25}$ when $T_{\text {dust }}$ decreases from $14 \mathrm{~K}$ to $12 \mathrm{~K}$.

To estimate the effect of the uncertainty related to the adopted emissivity index, $\beta$, and to allow direct comparison with some previous determinations of the dust opacity (e.g., Martin et al. 2012; Roy et al. 2013; Shirley et al. 2011), we repeated the analysis described above using fixed emissivity indices of $\beta=1.8$ and $\beta=2.2$. Lowering $\beta$ leads to higher dust colour temperatures, lower optical depths $\tau_{250}$, and lower values of $\sigma_{250 \mu \mathrm{m}}^{\mathrm{H}}$. The effect of increasing $\beta$ is the opposite. The values of $\sigma_{250 \mu \mathrm{m}}^{\mathrm{H}}$ and $\kappa_{250 \mu \mathrm{m}}^{\mathrm{g}}$ resulting from a linear fit to the $H-K_{\mathrm{s}}$ vs. $\tau_{250 \mu \mathrm{m}}$ correlation for $\beta=1.8$ and $\beta=2.2$ are the following:

$$
\begin{aligned}
\beta=1.8: \sigma_{250 \mu \mathrm{m}}^{\mathrm{H}} & =(1.5 \pm 0.3) \times 10^{-25} \mathrm{~cm}^{2} \mathrm{H}^{-1}, \\
\kappa_{250 \mu \mathrm{m}}^{\mathrm{g}} & =(0.06 \pm 0.01) \mathrm{cm}^{2} \mathrm{~g}^{-1}, \\
\beta=2.2: \sigma_{250 \mu \mathrm{m}}^{\mathrm{H}} & =(2.4 \pm 0.4) \times 10^{-25} \mathrm{~cm}^{2} \mathrm{H}^{-1}, \\
\kappa_{250 \mu \mathrm{m}}^{\mathrm{g}} & =(0.10 \pm 0.02) \mathrm{cm}^{2} \mathrm{~g}^{-1} .
\end{aligned}
$$

In both cases the extrapolated dust absorption cross-section per unit mass of gas at $\lambda=850 \mu \mathrm{m}$ happens to be the same as for $\beta=2.0$, i.e., $\kappa_{850 \mu \mathrm{m}}^{\mathrm{g}}=0.007 \mathrm{~cm}^{2} \mathrm{~g}^{-1}$. The values at $450 \mu \mathrm{m}$ change slightly depending on $\beta: \kappa_{450 \mu \mathrm{m}}^{\mathrm{g}}=0.022-0.028 \mathrm{~cm}^{2} \mathrm{~g}^{-1}$ for $\beta=1.8-2.2$. These results should be compared with predictions from various models listed in Table 2 of Shirley et al. (2005).

The suggested temperature dependence of the crosssection $\sigma_{250 \mu \mathrm{m}}^{\mathrm{H}}$ steepens with the adopted $\beta$. For $\beta=1.8$ the 
increase in $\sigma_{250 \mu \mathrm{m}}^{\mathrm{H}}$ is about $24 \%$ when $T_{\text {dust }}$ decreases from $14 \mathrm{~K}$ to $12 \mathrm{~K}$, whereas for $\beta=2.2$ the corresponding increase is $37 \%$.

\section{Discussion}

The extinction ratio $A_{250 \mu \mathrm{m}} / A_{J}=0.0014 \pm 0.0002$, and the implied values of the dust absorption cross-section, $\sigma_{250 \mu \mathrm{m}}^{\mathrm{H}}=$ $(1.8 \pm 0.3) \times 10^{-25} \mathrm{~cm}^{2} \mathrm{H}^{-1}$, and the opacity per unit mass of gas, $\kappa_{250 \mu \mathrm{m}}^{\mathrm{g}}=(0.08 \pm 0.01) \mathrm{cm}^{2} \mathrm{~g}^{-1}$, derived for the cold core CrA C are close to the widely adopted "standard" values from Hildebrand (1983). The derived absorption cross-section is similar or slightly smaller than the values derived previously for molecular clouds (e.g., Martin et al. 2012 and references therein). For example, Terebey et al. (2009) determined the opacity $\kappa_{160 \mu \mathrm{m}}=(0.23 \pm 0.05) \mathrm{cm}^{2} \mathrm{~g}^{-1}$ at $160 \mu \mathrm{m}$ (assuming $\beta=2.0$ ) for low extinction regions ( $A_{V}$ up to $4 \mathrm{mag}$ ) in the Taurus cloud L1521, when we get $\kappa_{160 \mu \mathrm{m}}^{\mathrm{g}}=(0.19 \pm 0.03) \mathrm{cm}^{2} \mathrm{~g}^{-1}$ in CrA C. Adopting the spectral emissivity index $\beta=1.8$ used in several previous studies, we derive a dust absorption crosssection of $\sigma_{250 \mu \mathrm{m}}^{\mathrm{H}}=(1.5 \pm 0.3) \times 10^{-25} \mathrm{~cm}^{2} \mathrm{H}^{-1}$. This value lies close to the low end of the range derived by Martin et al. (2012), using similar methods and the same $\beta$, towards the Vela cloud near the Galactic plane. We note that our $\sigma_{250 \mu \mathrm{m}}^{\mathrm{H}}$ has the same meaning as $\sigma_{\mathrm{e}}(1200)$, and that our $\kappa_{250 \mu \mathrm{m}}^{\mathrm{g}}$ is denoted by $r \kappa_{0}$ in Martin et al. Furthermore, we have adopted the colour excess to column density ratio from Martin et al. (2012). The $\tau_{250} / N_{\mathrm{H}}$ ratio (implying $\left.\sigma_{250 \mu \mathrm{m}}^{\mathrm{H}}=(2.3 \pm 0.3) \times 10^{-25} \mathrm{~cm}^{2} \mathrm{H}^{-1}\right)$, derived by Planck Collaboration (2011) for the "dense" component (regions detected in $\mathrm{CO}$ ) of the Taurus Molecular Cloud complex, is larger than the value obtained here assuming $\beta=1.8$.

A rough agreement is found with some previous determinatons of the dust opacity in dense cores. The value of $\sigma_{250 \mu \mathrm{m}}^{\mathrm{H}}$ we obtain is similar to that determined in the Thumbprint Nebula (Lehtinen et al. 1998), and midway the values derived towards two positions in LDN 1688 (Rawlings et al. 2013). Shirley et al. (2011) determined in the protostellar core B335 the opacity ratio $\kappa_{850} \mu \mathrm{m} / \kappa_{K} \sim(3.2-4.8) \times 10^{-4}$, which agrees with the ratio $\kappa_{850 \mu \mathrm{m}} / \kappa_{V} \sim 4 \times 10^{-5}$ derived by Bianchi et al. (2003) for the starless globule B68. The opacity ratios determined by Kramer et al. (2003) in the IC 5146 filament show more variety, $\kappa_{850 \mu \mathrm{m}} / \kappa_{V} \sim(1.3-5.0) \times 10^{-5}$, but there is a clear temperature dependence, and CrA C should be compared with the cool parts of IC 5146, i.e. the high end of Kramer's opacity range.

When compared with the frequently used dust model of Ossenkopf \& Henning (1994), our average dust opacity $\kappa_{850 \mu \mathrm{m}}^{\mathrm{g}}=$ $0.007 \mathrm{~cm}^{2} \mathrm{~g}^{-1}$ of gas $\left(\sim \kappa_{850 \mu \mathrm{m}}^{\mathrm{d}}=0.7 \mathrm{~cm}^{2} \mathrm{~g}^{-1}\right.$ of dust $)$ corresponds roughly to the opacity of unprocessed dust grains taken from the MRN (Mathis et al. 1977) size distribution, but lies clearly below the predictions for coagulated or/and ice-coated grains of the same model (see also the comparison between different models in Shirley et al. 2005).

In terms of data and methods used our study is similar to the recent work of Roy et al. (2013) who determined the dust opacities in the Orion A cloud using Herschel and 2MASS. The study of Roy et al. (2013) benefits from a large number of data points, majority of them corresponding to moderate extinctions $\left(A_{V}<8 \mathrm{mag}\right)$. The authors found indications of an increase in the dust opacity towards higher column densities, with $\left(\sigma_{\mathrm{FIR}}^{\mathrm{H}} / 10^{-25} \mathrm{~cm}^{2}\right)=\left(N_{\mathrm{H}} / 10^{21} \mathrm{~cm}^{-2}\right)^{0.28}$ (see their Fig. 4). In order to compare this relationship with the situation observed in $\mathrm{CrA} \mathrm{C}$, we have replotted in Fig. 7 the $H-K_{\mathrm{s}}$ versus

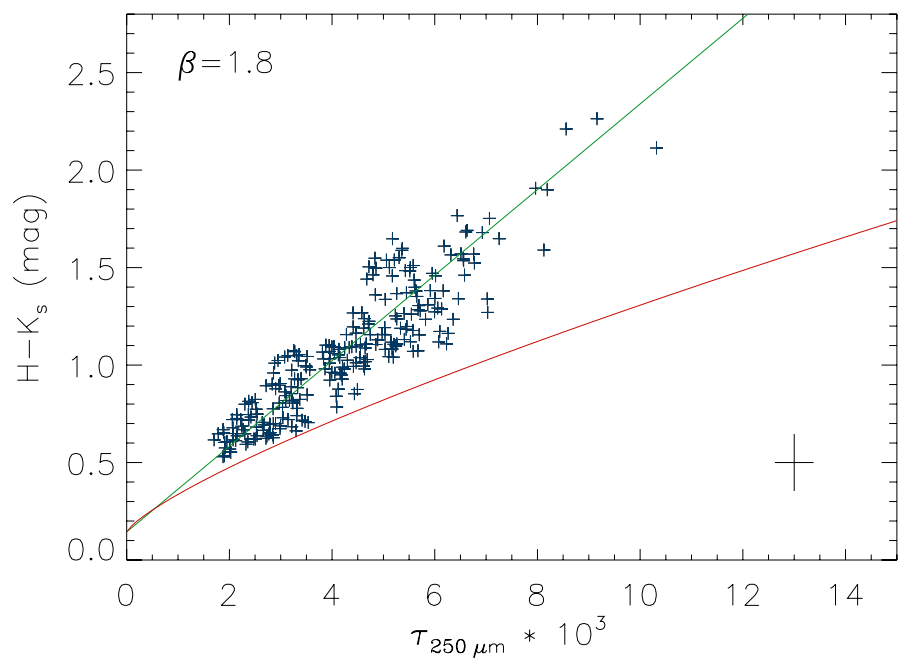

Fig. 7. $H-K$ colours of the background stars as a function $\tau_{250} \mu \mathrm{m}$ from Herschel assuming a dust emissivity index of $\beta=1.8$. A linear fit to the data points is shown with a green line. The red curve shows the relationship implied by the dependence of $\sigma_{\mathrm{FIR}}^{\mathrm{H}}$ on $N_{\mathrm{H}}$ found in Orion A by Roy et al. (2013). The mean error of the data points is indicated with a cross in the bottom right.

$\tau_{250 \mu \mathrm{m}}$ correlation derived using $\beta=1.8$ as assumed in Roy et al. (2013). In this figure, we have also plotted the relationship corresponding to the dependence of $\sigma_{\mathrm{FIR}}^{\mathrm{H}}$ on $N_{\mathrm{H}}$ found in Orion A, namely $H-K_{\mathrm{s}}=\left(H-K_{\mathrm{s}}\right)_{0}+42 \tau_{250 \mu \mathrm{m}}^{1 / 1.28}$. The change of the dust opacity towards higher column densities seems clearly less marked in CrAC than could have been expected on the basis of the results of Roy et al. (2013) in Orion.

The $A_{\mathrm{FIR}} / A_{J}$ vs. $T_{\mathrm{D}}$ correlation plot suggests, however, a change of $\sigma_{\mathrm{FIR}}^{\mathrm{H}}$ as a function of temperature (which in turn depends on the column density), and therefore a slight curvature can be present in the $H-K_{\mathrm{S}}$ vs. $\tau_{250 \mu \mathrm{m}}$ relationship. The slope of the $A_{\mathrm{FIR}} / A_{J}$ vs. $T_{\mathrm{D}}$ correlation is similar to that derived in L1642 by Lehtinen et al. (2007) for roughly the same temperature range as observed here, but clearly steeper than the gradient in IC 5146 reported by Kramer et al. (2003) (a factor of three increase in $A_{850 \mu \mathrm{m}} / A_{V}$ from $T_{\text {dust }}=20 \mathrm{~K}$ to $12 \mathrm{~K}$ ). The data of Lehtinen et al. suggest that the gradient in the $A_{\mathrm{FIR}} / A_{\mathrm{NIR}}$ ratio becomes smoother with increasing colour temperature. The implied increase in $\sigma_{\text {FIR }}^{\mathrm{H}}$ with a decreasing temperature possibly reflects the fact that in starless cores the highest densities are associated with the lowest dust temperatures. Monte Carlo simulations showed, however, that if the true relation is flat, noise tends to produce a strong negative correlation between the observed values of $A_{250 \mu \mathrm{m}} / A_{J}$ and $T_{\mathrm{d}}$. Assuming the $7 \%$ relative uncertainty for the surface brightness measurements, the slope is in the simulations negative and almost three times as large as the observed one. The $7 \%$ error estimate is very conservative for the band-to-band errors in Herschel data. Nevertheless, we clearly do not have strong evidence that $A_{250 \mu \mathrm{m}} / A_{J}$ is a decreasing function of temperature in CrA C.

Because of line-of-sight temperature variations, the fitted colour temperature overestimates the mass-averaged dust temperature (e.g., Shetty et al. 2009; Nielbock et al. 2012; Ysard et al. 2012). This leads to an underestimation of the optical depth, $\tau_{\lambda}$, and the dust opacity $\kappa_{\lambda}$ towards the densest regions (Juvela \& Ysard 2012; Malinen et al. 2011).

To estimate the importance of this effect in the present case, we examined a model that closely resembles the $\mathrm{CrA} \mathrm{C}$ core. The model is detailed in Appendix A. The modelling result 
suggests that indeed, the values of $\tau_{250 \mu \mathrm{m}}$ derived from the observations may underestimate the "true" optical depths of the model by $\sim 30 \%$ at the high end of the $H-K_{\mathrm{s}}$ versus $\tau$ correlation. In Appendix B we examine the change in the $H-K_{\mathrm{s}}$ versus $\tau_{250 \mu \mathrm{m}}$ correlation assuming that the relationship between the "observed" and "true" optical depth is the same as in the model. It turns out that the correlation plot $H-K_{\mathrm{s}}$ versus the "corrected" $\tau_{250 \mu \mathrm{m}}$ agrees quite well with the relationship implied by the dependence of $\sigma^{\mathrm{H}}$ on $N_{\mathrm{H}}$ determined by Roy et al. (2013), namely that $E\left(H-K_{\mathrm{s}}\right)=C \tau_{250 \mu \mathrm{m}}^{1 / 1.28}$. At the high end of the optical depth range examined here, the values of $\sigma_{250 \mu \mathrm{m}}^{\mathrm{H}}$ calculated from this relationship are $\sim 40 \%$ higher than obtained from the linear fit to the unmodified optical depths derived from observations.

To summarise, although we do not see direct evidence for changes of the dust opacity in our observations, it is likely that the dust temperature in the core decreases inward owing to the attenuation of the interstellar radiation field, and the optical depths, $\tau_{250 \mu \mathrm{m}}$, derived from observations underestimate the true values up to $30 \%$ near the core centre. The bias caused by difference between the colour temperature and the mass-averaged dust temperature was not corrected in the study of Roy et al. (2013), but probably the effect there was not as severe as in the present study because their data probe lower column density regions than that discussed here. Taking the bias into account, and adopting the emissivity exponent value $\beta=2.0$ we estimate that the dust absorption cross section per unit mass of gas, $\kappa_{250 \mu \mathrm{m}}^{\mathrm{g}}$, increases from $\sim 0.08 \mathrm{~cm}^{2} \mathrm{~g}^{-1}$ at the core edges to $\sim 0.11 \mathrm{~cm}^{2} \mathrm{~g}^{-1}$ close to the centre of the core. We note that the very nucleus of the core is not sampled in the present data because no stars were detected in this part.

The dust opacities are not particularly high compared with those found in dense clouds (e.g., Martin et al. 2012). In the cold cores examined by Juvela et al. (2011), the highest estimates were above $\kappa_{250 \mu \mathrm{m}}^{g}=0.2 \mathrm{~cm}^{2} \mathrm{~g}^{-1}$ but the values obtained for starless cores in Musca cloud were below $\kappa_{250 \mu \mathrm{m}}^{g} \sim 0.12 \mathrm{~cm}^{2} \mathrm{~g}^{-1}$ and thus similar to the values in CrA C. Owing to its location in the shielded "tail" of the CrA molecular cloud, near the centre of an extensive HI cloud (Llewellyn et al. 1981; Harju et al. 1993), the CrA C core is likely to be at an early stage of evolution. Previous molecular line observations support this idea. Firstly, several common high-density tracers, such as $\mathrm{N}_{2} \mathrm{H}^{+}, \mathrm{NH}_{3}$ and $\mathrm{H}^{13} \mathrm{CO}$, are weak in this core, suggesting that the gas has a moderate maximum density $\left(n_{\mathrm{H}_{2}} \sim 10^{5} \mathrm{~cm}^{-3}\right)$. This estimate is consistent with the cloud model constructed in Appendix A where the best fit to the Herschel far-infrared was obtained with a central density of $n_{\mathrm{H}}=1.5 \times 10^{5} \mathrm{~cm}^{-3}$. Secondly, $\mathrm{C}^{18} \mathrm{O}$ seems to be undepleted and peaks, together with the high-density tracers roughly at the same place as the thermal dust emission (Kontinen et al. 2003). As grain growth by accretion, and the corresponding depletion of abundant elements in the gas-phase need time to take effect, the relatively low value of $\kappa$ is perhaps connected with the low degree of molecular depletion derived in $\mathrm{CrA} \mathrm{C}$, both being indicators that suggest the relative youth of this core.

Acknowledgements. We thank Aurora Sicilia-Aguilar for providing the HAWK-I photometry of CrA, and Jean-Philippe Bernard for deriving the sky brightness offsets for the Herschel maps using Planck satellite data. Helpful discussions with Kalevi Mattila are thankfully acknowledged. This publication makes use of data products from the Two Micron All Sky Survey, which is a joint project of the University of Massachusetts and the Infrared Processing and Analysis Center/California Institute of Technology, funded by the National Aeronautics and Space Administration and the National Science Foundation. The study has been financially supported by the Academy of Finland through grants 127015, 132291, 140970 and 250741, and by the European Community FP7-ITN Marie-Curie Programme (grant agreement 238258).

\section{Appendix A: Examining dust temperature in a cloud model of CrA C}

The model cloud was discretised to $75^{3}$ cells, the projected area corresponding to $\sim 7^{\prime} \times 7^{\prime}$. The temperature distribution in the three-dimensional model was solved with Monte Carlo radiative transfer calculations, assuming the dust properties of Ossenkopf \& Henning (1994) (thin ice mantles accreted in $10^{5}$ years at a density of $10^{6}$ ). The predicted surface brightness was compared to the data on CrA C and the column density corresponding to each of the $75 \times 75$ map pixels was adjusted iteratively until the model exactly reproduced the $250 \mu \mathrm{m}$ observations. The external radiation field was selected so that also the shape of the model spectra and column density were similar to $\mathrm{CrA} \mathrm{C}$. To take into account the shielding by the surrounding cloud, the external field was first attenuated by an amount that corresponds to a dust layer with $A_{V}=1 \mathrm{mag}$. When the Mathis et al. (1983)
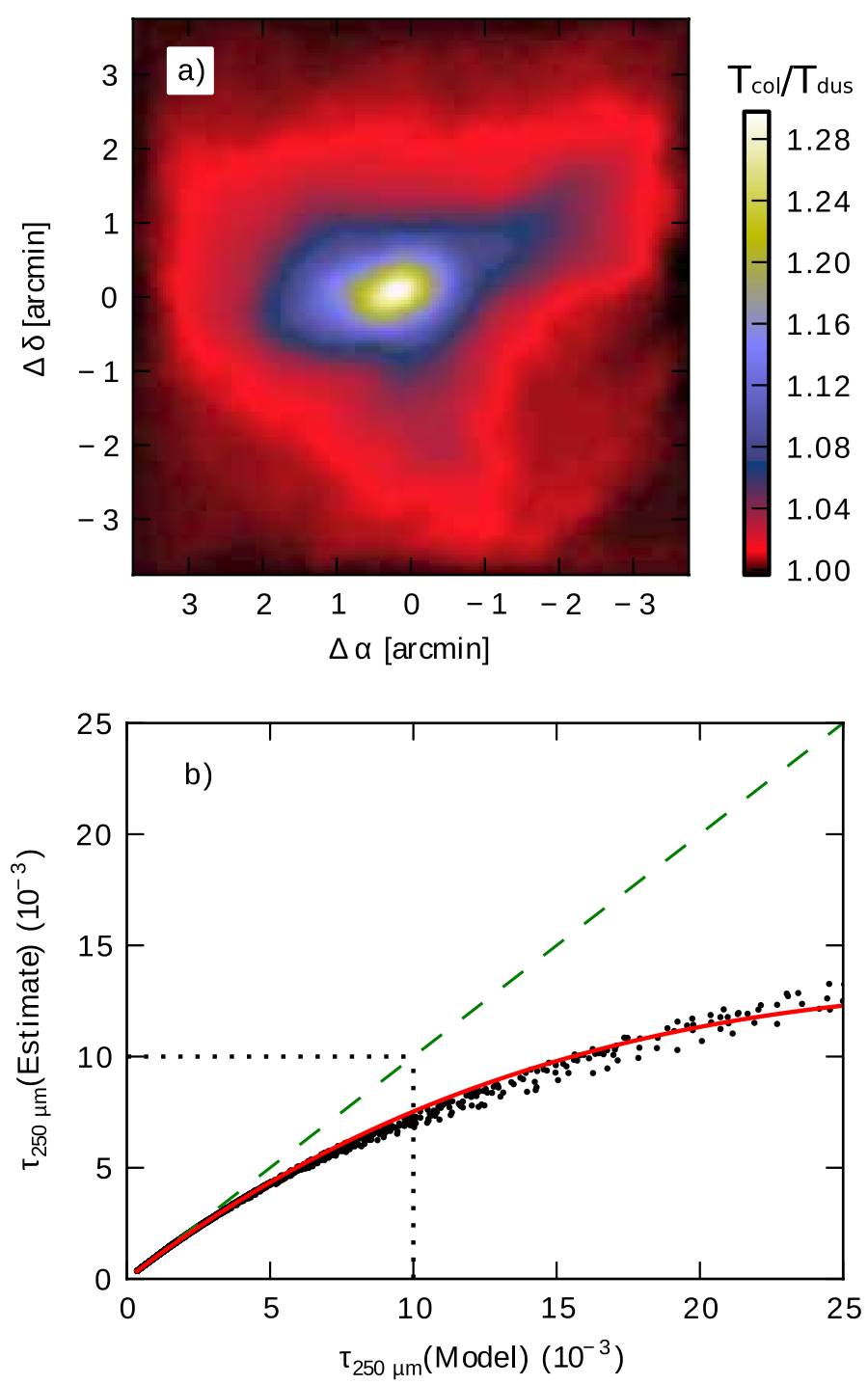

Fig. A.1. Results from a radiative transfer model resembling the CrA C core. Frame a) the ratio of the colour temperature (derived from synthetic surface brightness observations) and the mass-averaged real dust temperature. Frame b) the derived values of $\tau_{250 \mu \mathrm{m}}$ as the function of the true optical depth in the model. The dotted box corresponds to the range of optical depths in Fig. 7. The dashed green line represents a 1:1 correlation. The red curve represents an exponential function fitted to the data points (see the text). 
field was multiplied with a factor of 5.2, the central column density of the model was $\sim 30 \%$ higher than the estimated column density of $\mathrm{CrA} \mathrm{C}$ and the $160 \mu \mathrm{m}$ intensity was $\sim 10 \%$ above the observed.

The surface brightness maps of the model were analysed in the same way as the real observations, using $\beta=1.75$ applicable for the dust model. As expected, the column density derived from the surface brightness data was below the true values of the model, the error being more than a factor of two at the centre. This is illustrated in Fig. A.1 where the upper frame shows the ratio of the colour temperature and the mass-average dust temperature and the lower frame compares the estimated and the true values of $\tau_{250 \mu \mathrm{m}}$. The bias in the case of CrA C is, of course, not precisely known but can be of similar magnitude, i.e., up to $\sim 30 \%$ for the highest optical depths (column densities) considered in the $H-K$ vs. $\tau_{250 \mu \mathrm{m}}$ correlation where a similar $\beta$ has been assumed (Fig. 7). This would decrease the correlation slope and the reported $\sigma^{\mathrm{H}}$ and $\kappa_{250} \mu \mathrm{m}$ would need to be scaled up. Of course, similar biases may affect most of the values reported in the literature. In what follows we attempt to estimate the effect on $\sigma^{\mathrm{H}}$ in more detail.

\section{Appendix B: Dust absorption cross section using the modified optical depths}

The relationship between the "observed" and "true" optical depths in the model can be fitted with the function $\tau_{\text {obs }}=$ $\tau_{\text {true }} \mathrm{e}^{-b \tau_{\text {true }}}$, where $b=28.4$. In Fig. B.1, we have plotted again the observed $H-K$ colours in the background of CrA C but now against a modified $\tau_{250 \mu}$ m, assuming the same conversion between the observed and "true" values as in the model above. In this figure, we have also plotted the relationship $H-K_{\mathrm{s}}=$ $\left(H-K_{\mathrm{s}}\right)_{0}+C \tau_{250 \mu \mathrm{m}}^{1 / 1.28}$ implied by the finding of Roy et al. (2013) in Orion A that $\sigma^{\mathrm{H}} \propto N_{\mathrm{H}}^{0.28}$. A reasonable agreement with the data points is achieved by setting the constant $C=60$ instead of 42 which would make the curve bend down too strongly. This change is equivalent with making the coefficient of proportionality in the relationship $\sigma^{\mathrm{H}} \propto N_{\mathrm{H}}^{0.28}$ smaller than in Orion by a

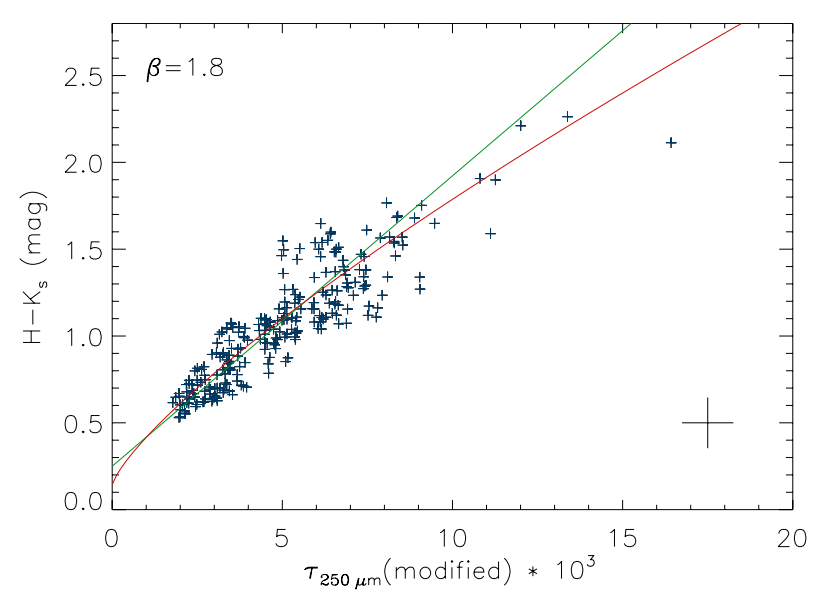

Fig. B.1. $H-K$ colours of stars in the background of $\mathrm{CrA} \mathrm{C}$ as a function of a modified $\tau_{250 \mu} \mu$ where we have attempted to correct for the effect of line-of-sight temperature variations. It is assumed that the relationship between the observed and "true" optical depth is similar to the model shown in Fig. A.1. The red curve shows the relationship expected from the result of Roy et al. (2013) where the $\sigma^{\mathrm{H}}$ increases as $N_{\mathrm{H}}^{0.28}$ and the green line is a linear fit to the data points. The mean error of the data points is indicated with a cross in the bottom right.

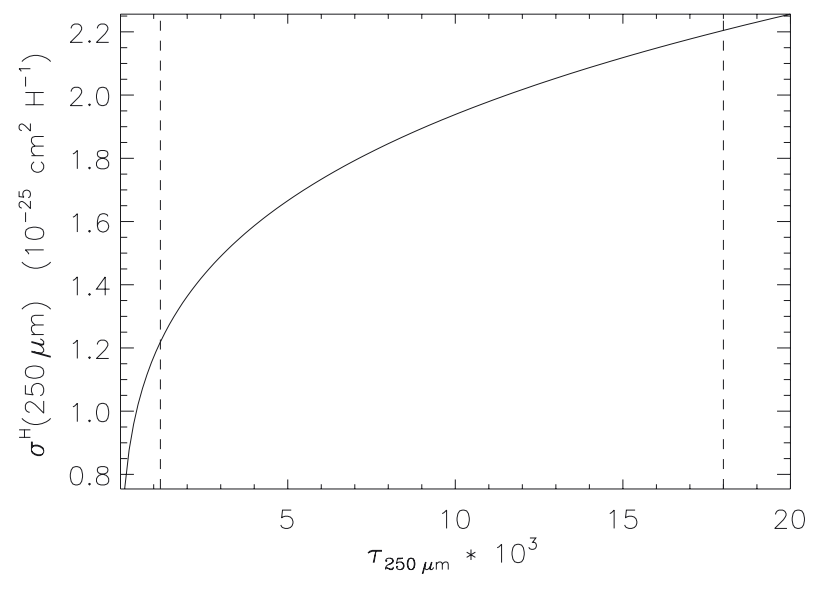

Fig. B.2. The dust absorption cross section $\sigma_{250 \mu \mathrm{m}}^{\mathrm{H}}$ as a function of $\tau_{250 \mu \mathrm{m}}$ as predicted by the empirical relationship between $\sigma^{\mathrm{H}}$ and $N_{\mathrm{H}}$ determined by Roy et al. (2013) in Orion A. The $\tau_{250} \mu \mathrm{m}$ range indicated with dashed lines corresponds to the range of "corrected" optical depths in $\mathrm{CrA} \mathrm{C}$ as estimated on grounds of our modeling results.

factor of $(42 / 60)^{1.28}=0.63$. The intersection with the $y$-axis is the same as in the linear fit, i.e $\left(H-K_{\mathrm{s}}\right)_{0}=0.14$. A linear fit to the data, $H-K_{\mathrm{s}}=(0.25 \pm 0.02)+(167 \pm 3) \tau_{250 \mathrm{~m} \text {,true }}$, is also shown in Fig. B.1. The linear fit gives a slightly larger $\chi^{2}$ than the curved relationship. Moreover, the intersection of the line with the $y$-axis, 0.25, is consistent with an M4 giant (Bessell \& Brett 1988) which seems less likely as a typical background star than a K3 giant.

Using the relationships $E\left(H-K_{\mathrm{S}}\right)=C \tau^{1 / 1.28}, \tau=N_{\mathrm{H}} \sigma^{\mathrm{H}}$, and $N_{\mathrm{H}}=E\left(H-K_{\mathrm{s}}\right) A(J) / E\left(H-K_{\mathrm{s}}\right) N_{\mathrm{H}} / A(J)$ one obtains the following expression for the dust absorption cross section as a funtion of the optical depth:

$\sigma_{250 \mu \mathrm{m}}^{\mathrm{H}}=\frac{\tau^{0.28 / 1.28}}{C A(J) / E(H-K) N_{\mathrm{H}} / A(J)}$.

The dust absorption cross section resulting from this formula is shown in Fig. B.2, for the range of (modified) $\tau_{250} \mu \mathrm{m}$ estimated for CrA C. Here we have assumed that $C=60$. The model suggests that $\sigma_{250 \mu \mathrm{m}}^{\mathrm{H}}$ increases from the value $1.2 \times 10^{-25} \mathrm{~cm}^{2} \mathrm{H}^{-1}$ to $2.2 \times 10^{-25} \mathrm{~cm}^{2} \mathrm{H}^{-1}$ at the largest optical depths, when the linear fit using the unmodified optical depths gave $\sigma_{250 \mu \mathrm{m}}^{\mathrm{H}}=$ $1.5 \times 10^{-25} \mathrm{~cm}^{2} \mathrm{H}^{-1}$.

\section{References}

André, P., Men'shchikov, A., Bontemps, S., et al. 2010, A\&A, 518, L102 Arab, H., Abergel, A., Habart, E., et al. 2012, A\&A, 541, A19

Ascenso, J., Alves, J., Beletsky, Y., \& Lago, M. T. V. T. 2007, A\&A, 466, 137

Bertin, E., \& Arnouts, S. 1996, A\&AS, 117, 393

Bessell, M. S., \& Brett, J. M. 1988, PASP, 100, 1134

Bianchi, S., Gonçalves, J., Albrecht, M., et al. 2003, A\&A, 399, L43

Bohlin, R. C., Savage, B. D., \& Drake, J. F. 1978, ApJ, 224, 132

Cardelli, J. A., Clayton, G. C., \& Mathis, J. S. 1989, ApJ, 345, 245

Chini, R., Kämpgen, K., Reipurth, B., et al. 2003, A\&A, 409, 235

Golay, M. 1974, Introduction to astronomical photometry, Astrophys. Space Sci. Lib., 41

Griffin, M. J., Abergel, A., Abreu, A., et al. 2010, A\&A, 518, L3

Harju, J., Haikala, L. K., Mattila, K., et al. 1993, A\&A, 278, 569

Hildebrand, R. H. 1983, QJRAS, 24, 267

Indebetouw, R., Mathis, J. S., Babler, B. L., et al. 2005, ApJ, 619, 931

Juvela, M., \& Ysard, N. 2012, A\&A, 539, A71

Juvela, M., Ristorcelli, I., Pelkonen, V.-M., et al. 2011, A\&A, 527, A111

Kontinen, S., Harju, J., Caselli, P., Heikkilä, A., \& Walmsley, M. 2003, in SFChem 2002: Chemistry as a Diagnostic of Star Formation, eds. C. L. Curry, \& M. Fich, 331 
Kramer, C., Richer, J., Mookerjea, B., Alves, J., \& Lada, C. 2003, A\&A, 399, 1073

Lada, C. J., Lada, E. A., Clemens, D. P., \& Bally, J. 1994, ApJ, 429, 694

Lehtinen, K., Lemke, D., Mattila, K., \& Haikala, L. K. 1998, A\&A, 333, 702

Lehtinen, K., Juvela, M., Mattila, K., Lemke, D., \& Russeil, D. 2007, A\&A, 466, 969

Li, A., \& Draine, B. T. 2001, ApJ, 554, 778

Llewellyn, R., Payne, P., Sakellis, S., \& Taylor, K. N. R. 1981, MNRAS, 196, 29P

Malinen, J., Juvela, M., Collins, D. C., Lunttila, T., \& Padoan, P. 2011, A\&A, 530, A101

Martin, P. G., Roy, A., Bontemps, S., et al. 2012, ApJ, 751, 28

Mathis, J. S. 1990, ARA\&A, 28, 37

Mathis, J. S., Rumpl, W., \& Nordsieck, K. H. 1977, ApJ, 217, 425

Mathis, J. S., Mezger, P. G., \& Panagia, N. 1983, A\&A, 128, 212

Nielbock, M., Launhardt, R., Steinacker, J., et al. 2012, A\&A, 547, A11

Ormel, C. W., Min, M., Tielens, A. G. G. M., Dominik, C., \& Paszun, D. 2011, A\&A, 532, A43

Ossenkopf, V., \& Henning, T. 1994, A\&A, 291, 943

Paradis, D., Bernard, J.-P., \& Mény, C. 2009, A\&A, 506, 745

Persson, S. E., Murphy, D. C., Krzeminski, W., Roth, M., \& Rieke, M. J. 1998, AJ, 116, 2475
Peterson, D. E., Caratti o Garatti, A., Bourke, T. L., et al. 2011, ApJS, 194, 43 Pilbratt, G. L., Riedinger, J. R., Passvogel, T., et al. 2010, A\&A, 518, L1

Planck Collaboration 2011, A\&A, 536, A25

Poglitsch, A., Waelkens, C., Geis, N., et al. 2010, A\&A, 518, L2

Rawlings, M. G., Juvela, M., Lehtinen, K., Mattila, K., \& Lemke, D. 2013, MNRAS, 428, 2617

Roussel, H. 2012 [arXiv: 1205.2576]

Roy, A., Martin, P. G., Polychroni, D., et al. 2013, ApJ, 763, 55

Shetty, R., Kauffmann, J., Schnee, S., Goodman, A. A., \& Ercolano, B. 2009, ApJ, 696, 2234

Shirley, Y. L., Nordhaus, M. K., Grcevich, J. M., et al. 2005, ApJ, 632, 982

Shirley, Y. L., Huard, T. L., Pontoppidan, K. M., et al. 2011, ApJ, 728, 143

Sicilia-Aguilar, A., Henning, T., Kainulainen, J., \& Roccatagliata, V. 2011, ApJ, 736, 137

Skrutskie, M. F., Cutri, R. M., Stiening, R., et al. 2006, AJ, 131, 1163

Stead, J. J., \& Hoare, M. G. 2009, MNRAS, 400, 731

Stepnik, B., Abergel, A., Bernard, J.-P., et al. 2003, A\&A, 398, 551

Straižys, V., \& Lazauskaite, R. 2008, Baltic Astron., 17, 277

Terebey, S., Fich, M., Noriega-Crespo, A., et al. 2009, ApJ, 696, 1918

Vuong, M. H., Montmerle, T., Grosso, N., et al. 2003, A\&A, 408, 581

Weingartner, J. C., \& Draine, B. T. 2001, ApJ, 548, 296

Ysard, N., Juvela, M., Demyk, K., et al. 2012, A\&A, 542, A21 